\title{
Health care transition in pediatric neurosurgery: a consensus statement from the American Society of Pediatric Neurosurgeons
}

\author{
Brandon G. Rocque, MD, ${ }^{1}$ Bradley E. Weprin, MD, ${ }^{2}$ Jeffrey P. Blount, MD, ${ }^{1}$ \\ Betsy D. Hopson, MSHA, ${ }^{1}$ James M. Drake, MBBCh, ${ }^{3}$ Mark G. Hamilton, MD, ${ }^{4}$ \\ Michael A. Williams, MD, ${ }^{5,6}$ Patience H. White, MD, MA, ${ }^{7}$ Katie O. Orrico, JD, ${ }^{8}$ and \\ Jonathan E. Martin, MD9
}

\begin{abstract}
'Department of Neurosurgery, University of Alabama at Birmingham, Children's of Alabama, Birmingham, Alabama; ${ }^{2}$ Department of Pediatric Neurosurgery, University of Texas Southwestern Medical Center, Dallas, Texas; ${ }^{3}$ Division of Neurosurgery, Hospital for Sick Children, Toronto, Ontario, Canada; ${ }^{2}$ Department of Clinical Neurosciences, University of Calgary, Alberta, Canada; Departments of ${ }^{5}$ Neurology and ${ }^{6}$ Neurological Surgery, University of Washington School of Medicine, Seattle, Washington; ${ }^{7}$ The National Alliance to Advance Adolescent Health and Got Transition, Washington, DC; ${ }^{8}$ American Association of Neurological Surgeons/Congress of Neurological Surgeons Washington Office, Washington, DC; and 'Department of Surgery, Division of Neurosurgery, Connecticut Children's Medical Center, Hartford, Connecticut
\end{abstract}

OBJECTIVE The number of children with complex medical conditions surviving to adulthood is increasing. A planned transition to adult care systems is essential to the health maintenance of these patients. Guidance has been established for the general health care transition (HCT) from adolescence to adulthood. No formal assessment of the performance of pediatric neurosurgeons in HCT has been previously performed. No "best practice" for this process in pediatric neurosurgery currently exists. The authors pursued two goals in this paper: 1) define the current state of HCT in pediatric neurosurgery through a survey of the membership of the American Society of Pediatric Neurosurgeons (ASPN) on current methods of HCT, and 2) develop leadership-endorsed best-practice guidelines for HCT from pediatric to adult neurosurgical health care.

METHODS Completion of the Current Assessment of Health Care Transition Activities survey was requested of 178 North American pediatric neurosurgeons by using a web-based questionnaire to capture HCT practices of the ASPN membership. The authors concurrently conducted a PubMed/MEDLINE-based literature review of HCT for young adults with special health care needs, surgical conditions, and/or neurological conditions for the period from 1990 to 2018. Selected articles were assembled and reviewed by subject matter experts and members of the ASPN Quality, Safety, and Advocacy Committee. Best-practice recommendations were developed and subjected to peer review by external expert groups.

RESULTS Seventy-six responses to the survey (43\%) were received, and 62 respondents (82\%) answered all 12 questions. Scores of 1 (lowest possible score) were recorded by nearly $60 \%$ of respondents on transition policy, by almost $70 \%$ on transition tracking, by $85 \%$ on transition readiness, by at least $40 \%$ on transition planning as well as transfer of care, and by $53 \%$ on transition completion. Average responses on all core elements were $<2$ on the established 4-point scale. Seven best-practice recommendations were developed and endorsed by the ASPN leadership.

CONCLUSIONS The majority of pediatric neurosurgeons have transition practices that are poor, do not meet the needs of patients and families, and should be improved. A structured approach to transition, local engagement with adult neurosurgical providers, and national partnerships between pediatric and adult neurosurgery organizations are suggested to address current gaps in HCT for patients served by pediatric neurosurgeons.

https://thejns.org/doi/abs/10.3171/2019.12.PEDS19524

KEYWORDS health care transition; pediatric neurosurgery; American Society of Pediatric Neurosurgeons; survey; Got Transition 
I $\mathrm{T}$ is the goal of all pediatric physicians to improve the health of children. It logically follows that these children will mature into young adults who must assimilate into the adult health care system. It is estimated that around 1.1 million US youth with special health care needs turn 18 each year. ${ }^{14}$ "Health care transition" (HCT) is defined as the process of moving from a pediatric to an adult model of health care with or without transfer to a new clinician. This is often a time of great stress for the patient, the patient's family, and the practicing physician. The goals of transition are as follows: 1) to improve the ability of youth or young adults to manage their own health and effectively use health services, and 2) to have an organized clinical process in pediatric and adult practices to facilitate transition preparation, transfer of care, and integration into adult-centered care. However, providers are often ill-equipped to deal with both psychological and medical aspects of care for the transitioning patient, and patients are reluctant to leave health systems and care teams with which they are familiar. The result is often neglect of transition efforts; in a recent report, only $17 \%$ of parents believed that their adolescent with special health care needs received adequate transition preparation, often with unfortunate results. ${ }^{14} \mathrm{~A}$ poorly executed transition is associated with 1) poor health outcomes, including decreased treatment adherence, increased health care utilization, and increased morbidity; 2) psychological stress for both patient and family; and 3) increased costs. ${ }^{6,15}$

In 2017, the Hydrocephalus Association convened a summit on HCT for patients with hydrocephalus to raise a call to action regarding the need for structured HCT for their membership. In a 2018 joint statement, the American Academy of Pediatrics (AAP), American Academy of Family Physicians (AAFP), and American College of Physicians (ACP) reaffirmed previously established goals and approaches to transition and called for the use of the Six Core Elements of Health Care Transition. ${ }^{24}$ Also, in 2018, a survey of the membership of the American Society of Pediatric Neurosurgeons (ASPN) identified HCT as the highest priority topic for its advocacy efforts. The ASPN leadership tasked us with the following: 1) describe the current state of transitional care in pediatric neurosurgery, 2) provide best-practice recommendations for HCT in pediatric neurosurgery, and 3) encourage future efforts by pediatric neurosurgeons in the development of HCT programs specific to our patient population, including research on best practices for HCT for neurosurgical patients.

\section{Methods}

\section{Assessing the Current State of HCT in Pediatric Neurosurgery}

We conducted a survey of pediatric neurosurgeons to determine the status of HCT implementation at pediatric neurosurgical programs across North America. Using a survey administration tool (SurveyMonkey), we sent a questionnaire (described below) via email to all members of the ASPN. The ASPN consists of 178 active members, including the majority of pediatric neurosurgeons in
North America who are certified by the American Board of Pediatric Neurological Surgery. No financial incentive for survey completion was offered, and no follow-up emails were sent to request survey completion. Verbal follow-up was announced to attendees of the 2019 ASPN annual meeting, where leadership encouraged completion of the previously emailed survey. Results were tabulated, analyzed, and used to identify topics for further discussion at the meeting, with the ultimate goal of developing an advocacy program on HCT.

Survey questions were based on questions 1-6 of the Current Assessment of Health Care Transition Activities, provided by Got Transition, a federally funded effort by the Maternal and Child Health Bureau and The National Alliance to Advance Adolescent Health. ${ }^{12}$ Got Transition establishes a quality improvement method of transition planning known as the Six Core Elements approach. These elements guide health care practices in developing and optimizing a transition process (Fig. 1). Survey questions aimed to determine the extent to which individual physicians or practices adhere to the Got Transition program. Seven additional questions assessed the respondents' demographics and practice type. This research was retrospectively reviewed by the Connecticut Children's Institutional Review Board and deemed exempt from a requirement of approval.

\section{Best-Practice Development}

At the annual meeting of the ASPN in 2019, a panel composed of subject matter experts and members of the ASPN Quality, Safety, and Advocacy Committee was convened. A literature review of PubMed/MEDLINE databases for the period from 1990 to 2018 was conducted to identify relevant material on HCT for young adults with special health care needs, surgical conditions, and/or neurological conditions. A position statement was developed and reviewed by external reviewers from Got Transition and the Hydrocephalus Association. A revised document was approved for dissemination by the ASPN leadership in July 2019.

\section{Results \\ Survey Results}

Questions 1-6 of the Current Assessment of Health Care Transition Activities survey were emailed to 178 North American pediatric neurosurgeons. Seventy-six responses $(43 \%)$ were received, and 62 respondents $(82 \%)$ answered all 12 questions. Information about respondents' age, sex, practice type, and geographic region are listed in Table 1.

In addition to the 6 demographic questions, the survey included 6 questions on transition (Table 2), each assessing the degree to which the respondent's practice met one of the Six Core Elements of transition as defined by the Got Transition program. Survey questions assessed the degree of preparation of the respondent's practice/department on a scale of 1-4; a score of 1 represented the least degree of preparation, whereas a score of 4 represented a welldeveloped transition processes. The Six Core Elements of transition were evaluated as follows: 


\section{Six Core Elements ${ }^{\mathrm{TM}}$ Approach for Youth Transitioning to Adult Health Care}

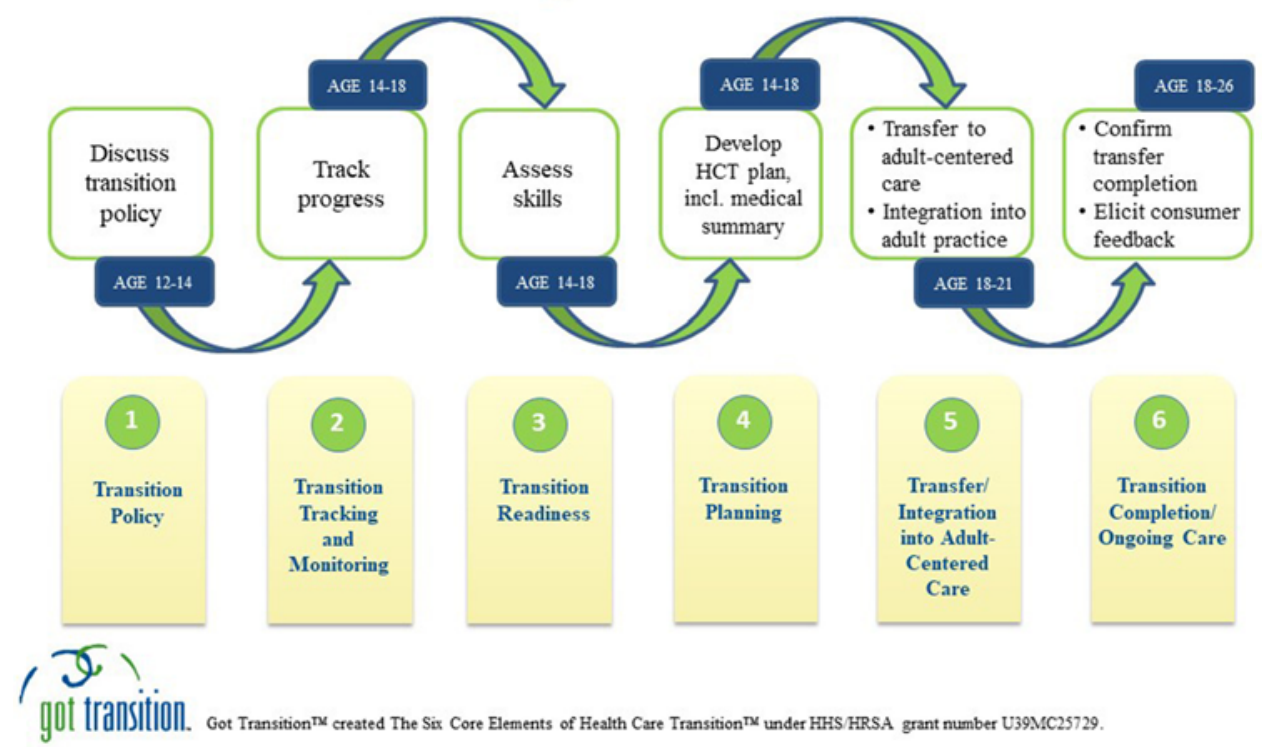

FIG. 1. The Six Core Elements of transition and their timing in clinical practice. The Six Core Elements of Health Care Transition are the copyright of Got Transition. This version of the Six Core Elements has been modified and is used with permission. incl. = including. Figure is available in color online only.

1) Transition policy: Does the respondent's practice/department have a defined policy for transition, was it written, and was it routinely shared with patients and families?

2) Transition tracking and monitoring: Does the respondent's practice/department maintain a registry of patients aged 14 and older?

3) Transition readiness: Does the respondent's practice/ department offer time alone (when cognitively able/ appropriate) with youth aged 14 or older and use standardized tools to assess transition readiness?

4) Transition planning: Does the respondent's practice/ department incorporate transition into a plan of care template for all patients aged 12 or older to include decision-making supports and a vetted list of adult care providers?

5) Transfer of care: Does the respondent's practice/department send a completed transfer package to a selected adult care provider?

6) Transfer completion: Does the respondent's practice/ department confirm transfer completion with feedback from the patient regarding transition to allow for process improvement?

Transition practices of ASPN members were poor in all 6 of the core elements assessed using this survey (Table 3 and Fig. 2). Scores of 1 (lowest possible score) were recorded by nearly $60 \%$ of respondents on transition policy, by almost $70 \%$ on transition tracking, by $85 \%$ on transition readiness, by at least $40 \%$ on transition planning as well as transfer of care, and by 53\% on transition completion. Average responses on all core elements were $<2$ on the established 4-point scale. Individual scores of 3 or 4 were very rare, with fewer than $10 \%$ scoring either 3 or higher in 5 of the 6 survey questions.

\section{Discussion}

"Health care transition" refers to the planned transfer and integration of adolescents/young adults from the pediatric health care system to the adult health care system. A systematic review has shown that a structured transition process improves population health (adherence, improved quality of life), experience of care (patient satisfaction), and utilization (increase in adult outpatient visits, decrease in emergency department visits and hospitalizations). ${ }^{11,15}$ In addition to preparing young adults and their families for the transition to adult care, a crucial component of successful transition is the assurance of equivalent care in an adult environment, particularly for children with medical complexity. Construction of a safe and mutually agreeable transition from pediatric to adult care providers (when required) remains one of the most challenging aspects of HCT in neurosurgery.

A recent report from the Hydrocephalus Association Transition Summit identified a number of barriers to successful HCT. ${ }^{25}$ Patients and their families present challenges, which include poor compliance/limited selfmanagement or "hovering" behaviors of parents with limited opportunities for patient independence. Health care systems can also encumber transition through absent or inadequate health care coverage for chronic complex conditions and the lack of models for successful transition. Of paramount importance is the lack of adult and pediatric 
TABLE 1. Demographics of survey respondents

\begin{tabular}{lc}
\hline \multicolumn{1}{c}{ Variable } & Percentage \\
\hline Sex & \\
\hline Male & $82 \%$ \\
\hline Female & $18 \%$ \\
\hline Age in yrs & \\
\hline $40-44$ & $9 \%$ \\
\hline $45-54$ & $39 \%$ \\
\hline $55-64$ & $36 \%$ \\
\hline $65+$ & $16 \%$ \\
\hline Yrs in practice since training & \\
\hline$<5$ & $2 \%$ \\
\hline $5-10$ & $7 \%$ \\
\hline $11-20$ & $39 \%$ \\
\hline $21-30$ & $36 \%$ \\
\hline $31+$ & $16 \%$ \\
\hline Practice structure & \\
\hline Full-time academic & $71 \%$ \\
\hline Private & $5 \%$ \\
\hline Hybrid & $13 \%$ \\
\hline Hospital employee & $9 \%$ \\
\hline Other & $21 \%$ \\
\hline Practice setting & $27 \%$ \\
\hline Large multispecialty group & $71 \%$ \\
\hline Small single-specialty group (2-5) & $9 \%$ \\
\hline Medium single-specialty group (6-20) & \\
\hline Large single-specialty group (>20) & $2 \% \%$ \\
\hline Other & \\
\hline Geographic region & \\
\hline New England/Mid-Atlantic & \\
\hline South Atlantic & \\
\hline Central & \\
\hline Mountain/Southwest & \\
\hline Pacific & \\
\hline
\end{tabular}

health care professionals who are well prepared to execute a successful transition for these patients.

The present survey of the ASPN membership illuminates the current state of HCT among pediatric neurosurgeons in North America. The majority of pediatric neurosurgeons in this survey reported transition practices that are poor, do not meet the needs of patients and families, and should be improved. There are both ethical and economic arguments for addressing this gap. ${ }^{6}$ Vinchon and Dhellemmes reported on a small cohort of adult patients who had tumors, hydrocephalus, or dysraphism arising from childhood with regard to mortality, employment, and other social outcomes. ${ }^{23}$ These authors found nearly ubiquitous continued medical complexity and high rates of delayed complications from the primary illness. Gabriel et al. reviewed outcomes in pediatric to adult transition interventions from the perspective of population health, consumer experience, and utilization/cost of care. Nearly two-thirds of papers meeting their inclusion criteria demonstrated positive outcome measures in these 3 domains. ${ }^{11}$ In a recent cost-benefit analysis of a transitional care program in neurosurgery, the authors demonstrated a $16.3 \%$ reduction in length of stay and a $3.1 \%$ decrease in the readmission rate for enrolled patients. ${ }^{15}$ The benefits for medically complex patients are likely higher.

In some health care systems, pediatric neurosurgeons do not practice within adult health care facilities in their community. In such circumstances, it is crucial to identify adult neurosurgeons who are prepared, resourced, and willing to deliver care to young adults with pediatric neurosurgical conditions. The ASPN recognizes that organized neurosurgery must play a role in addressing the inadequate availability of adult care surgeons with expertise, resources, and the willingness to care for patients with pediatric diseases.

\section{Best-Practice Recommendations for HCT in Pediatric Neurosurgery}

Based on the above analysis of survey responses, literature review, and critical review by ASPN leadership, we make the following best-practice recommendations.

1) Pediatric neurosurgeons should review their current practice, select a transition model that is applicable to their practice, and explore and/or develop relationships to make the process actionable. Practices should develop the best model to incorporate the desired HCT process for their practice.

It is essential for pediatric neurosurgeons to recognize the importance of HCT for their adolescent patients. We recommend review of the available Six Core Element packages from Got Transition. ${ }^{12}$ Got Transition includes a framework for transition outlined in three customizable packages for use by pediatric, family medicine, internal medicine-pediatrics, and adult medicine practices: a) transitioning youth to an adult health care provider, b) integrating young adults into adult health care, and c) transitioning to an adult approach to health care without changing providers. By reviewing these materials, a pediatric neurosurgeon or neurosurgical practice can customize a transition process specific to their needs and the needs of patients.

2) Pediatric neurosurgeons should develop a written transition policy for their practice.

A written transition policy is the cornerstone of any transition process, and there are several key elements to an effective policy. a) The transition policy should make clear the age at which the transition process begins. The AAP/AAFP/ACP clinical report recommends introducing the concept of transition by age $12 .{ }^{24} \mathrm{~b}$ ) The policy should include information about future visits in which the provider will spend time with the adolescent patient alone (when cognitively able or appropriate), without the parent present. Policies should take into account state laws about the age of majority (age 18 in most US states) and address access to information and health care decision-making for parents of children who have attained that age. For children who may have limited intellectual capacity, this is the first step in ensuring that the proper legal procedures are 


\section{TABLE 2. Questionnaire for advocacy survey}

Q1: Transition policy

1. Clinicians vary in their approach to health care transition, including the appropriate age for transfer to adult providers.

2. Clinicians follow a uniform but not a written policy about the age for transfer. The approach for transition planning differs among clinicians.

3. The practice has a written transition policy or approach, developed with input from youth and families that includes privacy and consent information and addresses the practice's transition approach and age of transfer. The policy is not consistently shared with youth and families.

4. The practice has a written transition policy or approach, developed with input from youth and families that includes privacy and consent information, a description of the practice's approach to transition, and age of transfer. Clinicians discuss it with youth and families beginning at ages 12 to 14 . The policy is publicly posted and familiar to all staff.

\section{Q2: Transition tracking \& monitoring}

1. Clinicians vary in the identification of transitioning youth, but most wait until close to the age of transfer to identify and prepare youth.

2. Clinicians use patient records to document certain relevant transition information (e.g., future provider information, date of transfer).

3. The practice has an individual transition flow sheet or registry for identifying and tracking transitioning youth, ages 14 and older, or a subgroup of youth with chronic conditions as they progress through and complete some but not all transition processes.

4. The practice has an individual transition flow sheet or registry for identifying and tracking transitioning youth, ages 14 and older, or a subgroup of youth with chronic conditions as they progress through and complete all "Six Core Elements of Health Care Transition 2.0," using EHR if possible.

\section{Q3: Transition readiness}

1. Clinicians vary in terms of the age when youth begin to have time alone during preventive visits without the parent/caregiver present. Transition readiness is seldom assessed.

2. Clinicians consistently offer time alone for youth after age 14 during preventive visits without the parent/caregiver present. They usually wait to assess transition readiness/self-care skills close to the time of transfer.

3. The practice consistently offers clinician time alone with youth after age 14 with clinicians during preventive visits, and clinicians discuss transition readiness/self-care skills and changes in adult-centered care beginning at ages 14 to 16 , but no formal assessment tool is used.

4. The practice consistently offers clinician time alone with youth after age 14 during preventive visits. Clinicians use a standardized transition readiness assessment tool. Self-care needs and goals are incorporated into the youth's plan of care beginning at ages 14 to 16 .

\section{Q4: Transition planning}

1. Clinicians vary in addressing health care transition needs and goals. They seldom make available a plan of care (including medical summary and emergency care plan and transition goals and action steps) or a list of adult providers.

2. Clinicians consistently address transition needs and goals as part of the plan of care. They usually provide a list of adult providers close to the time of transfer.

3. The practice partners with youth and families in developing and updating their plan of care with prioritized transition goals and preferences for securing an adult provider. This plan of care is regularly updated and accessible to youth and families.

4. The practice has incorporated transition into its plan of care template for all patients. All clinicians are encouraged to partner with youth and families in developing transition goals and updating and sharing the plan of care. Clinicians address needs for decision-making supports prior to age 18. The practice has a vetted list of adult providers and assists youth in identifying adult providers.

Q5: Transfer of care

1. Clinicians usually send medical records to adult providers in response to transitioning patient requests.

2. Clinicians consistently send medical records to adult providers for their transitioning patients.

3. The practice sends a transfer package that includes the plan of care (including the latest transition readiness assessment, transition goals/actions, medical summary and emergency care plan, and, if needed, legal documents, and a condition fact sheet).

4. The practice sends a complete transfer package (including the latest transition readiness assessment, transition goals/actions, medical summary and emergency care plan, and, if needed, legal documents, and a condition fact sheet), and pediatric clinicians communicate with adult clinicians, confirming pediatric provider's responsibility for care until young adult is seen in the adult practice.

Q6: Transition completion

1. Clinicians have no formal process for follow-up with patients who have transferred to new adult providers.

2. Clinicians encourage patients to let them know whether or not the transfer to new adult provider went smoothly.

3. The pediatric practice communicates with the adult practice confirming completion of transfer/first appointment and offering consultation assistance, if needed.

4. The practice confirms transfer completion, need for consultation assistance, and elicits feedback from patients regarding the transition experience.

$E H R=$ electronic health record; $Q=$ question.

Respondents were asked to choose the option in each category that best described their current practice in 6 areas. 
TABLE 3. Survey results with number of respondents in each category by question

\begin{tabular}{ccccccc}
\hline Level & Q1 & Q2 & Q3 & Q4 & Q5 & Q6 \\
\hline 1 & $39(57 \%)$ & $43(69 \%)$ & $51(85 \%)$ & $24(41 \%)$ & $25(43 \%)$ & $30(53 \%)$ \\
\hline 2 & $21(31 \%)$ & $15(24 \%)$ & $4(7 \%)$ & $27(47 \%)$ & $27(47 \%)$ & $14(25 \%)$ \\
\hline 3 & $5(7 \%)$ & $3(5 \%)$ & $3(5 \%)$ & $4(7 \%)$ & $4(7 \%)$ & $10(18 \%)$ \\
\hline 4 & $3(4 \%)$ & $1(2 \%)$ & $2(3 \%)$ & $3(5 \%)$ & $2(3 \%)$ & $3(5 \%)$ \\
\hline Total responses & 68 & 62 & 60 & 58 & 58 & 57 \\
\hline
\end{tabular}

followed for establishing parents or caregivers as guardians by the time of legal majority. c) The policy should clarify the process of transition and services offered by the practice and whether the youth/young adult will remain with the same provider, who will begin to offer an adult care model, or will transfer to an adult care provider. d) Finally, the policy should clearly state the age at which transition activities should be complete, and transfer to adult neurosurgical care should be achieved.

We recommend that a pediatric neurosurgical transition policy be readily accessible and at an appropriate reading level, whether through brochures and printed materials, online materials, or both, and that the policy be available in the languages that are common for patients of the practice. We recommend that neurosurgeons offer families the transition policy on a regular basis as they work through the transition process.

3) Pediatric neurosurgeons should develop a practice- specific process for HCT monitoring and assessment of readiness.

This process should include a review of the transition policy with patients and families prior to age 14 and at regular intervals thereafter. Each practice should have a system in place for tracking and monitoring participation in the transition program and progress toward adult health care. Transition monitoring systems may utilize the electronic medical record or be established as a separate database.

Each practice should establish a method for assessing transition readiness by using existing or other readiness tools. ${ }^{3,12}$ Pediatric neurosurgeons should determine which readiness assessment tool is most appropriate for their practice. Educational materials on practice-relevant medical issues should be available to review with patients and families. ${ }^{13,22}$ Assessments should include a determination of the youth's understanding of his or her medical condition (when cognitively able or appropriate), their ability to man-

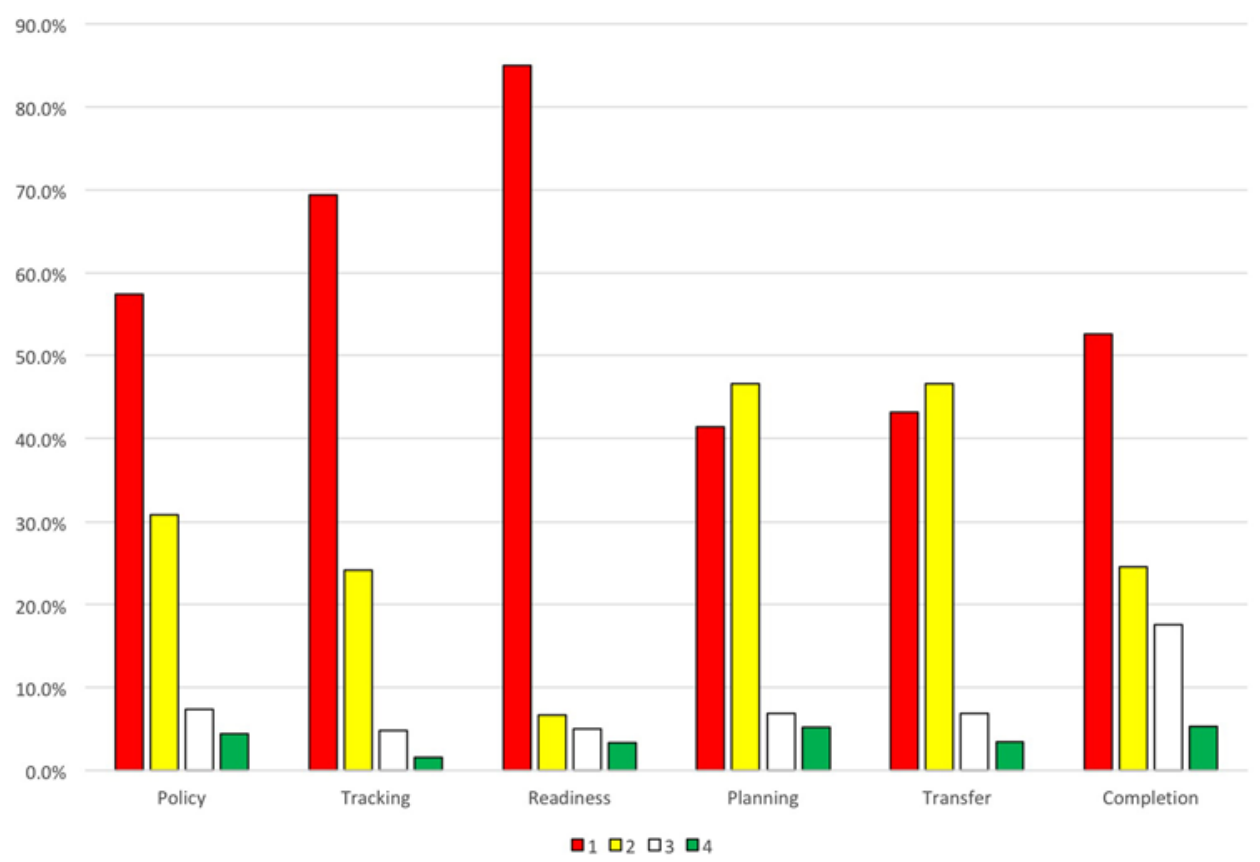

FIG. 2. Transition readiness of ASPN membership. Graphic representation of results of the survey questions from Fig. 1 to ASPN membership. Responses of 1 represent poor preparation for transition (red bar), whereas responses of 4 represent good preparation for transition (green bar). Figure is available in color online only. 
age their medical condition, their understanding of adult models of care, and their preparedness for interacting with the adult care system. The assessment should be administered at regular intervals to each transition-age patient.

4) Pediatric neurosurgeons should participate in the assessment of their patients' potential future decisionmaking capacity and legal competency.

Because a significant proportion of pediatric neurosurgical conditions have the potential to result in cognitive impairment, it is essential for health care providers to consider the patient's decision-making capacity when formulating a transition plan. The process of establishing legal guardianship or other supported decision-making options can be time-consuming and expensive. Discussions about decision-making capacity should be initiated early in the transition process to facilitate the completion of paperwork, legal filings, etc. Social workers and other support services can assist with this process. When the capacity for legal decision-making is unclear, formal assessments are available. ${ }^{4}$ Of course, decision-making capacity can evolve over time with neurosurgical conditions, improving or worsening. Therefore, regular reassessment is recommended, even if done only informally.

5) Pediatric neurosurgeons should engage with primary care and the entire health care team to develop the patient's transition plan and should contribute the neurosurgical component of the transition plan.

Children with neurosurgical conditions often have other medical needs. Even children who are otherwise healthy will have a pediatrician managing their medical care. The pediatric neurosurgical practice should establish a means of addressing short-term patient needs through the use of individual transition plans and long-term needs by contributing to the patient's comprehensive transitional care summary. Ideally, the pediatric neurosurgeon should identify the physician or practice that is in charge of the transition process, ensure that a comprehensive transition plan exists, and make certain that the family and child are aware of it. At a minimum, pediatric neurosurgeons should ensure that the neurosurgical components of the transition plan are completed and kept up to date. A method of communicating to the primary provider any changes in the neurosurgical condition or component of the transition plan should be established. Neuroimaging constitutes a unique component of transition for the patient under a neurosurgeon's care. Facilitation of image transfer to accepting providers/facilities or the provision of key images on transition summaries to the patient is a potential solution, depending on the diagnosis and circumstances.

6) Pediatric neurosurgeons should identify or assist the child and/or family in identifying an appropriate adult care provider for their neurosurgical condition. Pediatric neurosurgeons should request feedback on the existing transition process for the purpose of process improvement.

When transfer to an adult care provider is required, the pediatric neurosurgeon should assist with identifying an adult provider for neurosurgical care. This is among the most challenging aspects of neurosurgical care transition; in many areas, adult care providers are unable or unwilling to accept such patients in transition. Efforts to build trust and to work with adult care colleagues to improve comfort and competency in the treatment of these patients are essential elements of transition success. Once a successful working relationship is established, HCT should be an active process for each patient. This includes communication with the adult care neurosurgeon using standardized communication tools and the transfer of images or materials that will be necessary for the young adult's care. Best practice for adult care providers is an initial face-to-face meeting with the transitioning patient to clearly define the model of care, highlighting differences between adult and pediatric models of care.

Transfer of care providers can be one of the most trying times for families and youth who are accustomed to provider continuity and care in the pediatric setting. Identifying an adult care provider in advance of the transition is useful in helping to reduce anxiety and ensuring a smooth transfer. The transfer plan should also include a summary of past care, the most recent readiness assessment, and clear instructions about what to do in case of a medical emergency during the transfer period. Emergency instructions should include which provider to call and where to go for emergency medical services. Plans for emergency care and the completion of transfer to adult care should be clearly documented in the medical record.

Completion of the transfer process should also involve feedback from the patient and family about the existing transfer process. Got Transition provides feedback tools that can be customized to obtain this input. Regularly obtaining feedback will allow practices to refine and improve their transition process, working toward an HCT that is optimal for all involved.

7) Organized pediatric neurosurgery should continuously assess and update best practices and foster innovation in transitional care.

Such efforts should include the following: a) optimize infrastructure and payment models required for a successful pediatric neurosurgery transitional program, b) encourage research to demonstrate the positive financial impact and improved patient outcomes of a successful transitional program, c) promote education allowing for the development of new pediatric neurosurgery transitional care programs, and d) sponsor and foster discussion and innovation in the development and sustainment of transitional care programs in North America.

Access to and the availability of adult neurosurgical care remain difficult for many young adults with neurosurgical conditions. ${ }^{18,19,21}$ This remains a significant source of anxiety for patients and families. ${ }^{25}$ The ASPN is committed to working with adult neurosurgery organizations to ensure awareness of the needs of transitioning pediatric neurosurgical patients. Other chronic pediatric conditions, such as cystic fibrosis, have had success in improving adult outcomes through concerted efforts to improve transitional care and adult care. Such efforts can serve as models for progress in neurosurgery. ${ }^{1,2,5,8,10,17,20}$

\section{Study Limitations}

This study has several limitations. While a response rate of $43 \%$ and a completion rate of $35 \%$ are reasonable for an email survey completed by subspecialty providers, 
the potential for nonresponse bias remains. ${ }^{79}$ Survey administration favored completion by meeting attendees; no follow-up email contact or financial incentives were offered to increase survey completion by membership that did not attend the 2019 ASPN annual meeting. However, given the experience with similar surveys, this effort likely represents transitional care practices of the ASPN membership. ${ }^{16}$

While the implementation of transitional care programs in the practice of pediatric neurosurgery seems both indicated and desirable, operationalization of these efforts may be problematic in a surgical subspecialty with a limited capacity to see patients for nonsurgical health care maintenance visits and insufficient infrastructure to support the involved administrative requirements. The optimal interval between asymptomatic patient visits remains to be determined for pediatric neurosurgical patients. The pursuit of block grant funding or other financial support may be necessary to acquire adequate resources to support institutional programs. Advocacy and support of membership by organized pediatric neurosurgery are likely to be required for widespread adoption of transitional care programs in North America.

\section{Conclusions}

The majority of pediatric neurosurgeons have transition practices that are poor and do not meet the needs of patients and families. A structured approach to transition, local engagement with adult neurosurgical providers, and national partnerships between pediatric and adult neurosurgery organizations will be required to address current gaps in care. We suggest a methodology based on the Six Core Elements developed by The National Alliance to Advance Adolescent Health in order to improve HCT efforts for our patients. Partnership between pediatric and adult neurosurgery organizations will be necessary to address any gaps in HCT for adolescent patients from pediatric to adult health care systems. The ASPN is committed to the innovation, development, and sustainment of best practices in HCT for the patients and families we serve.

\section{References}

1. Abraham BP, Kahn SA: Transition of care in inflammatory bowel disease. Gastroenterol Hepatol (N Y) 10:633-640, 2014

2. Akre C, Suris JC, Belot A, Couret M, Dang TT, Duquesne A, et al: Building a transitional care checklist in rheumatology: a Delphi-like survey. Joint Bone Spine 85:435-440, 2018

3. American College of Physicians: ACP Pediatric to Adult Care Transitions Initiative. Philadelphia: American College of Physicians, 2019 (https://www.acponline.org/ clinical-information/high-value-care/resources-for-clinicians/ pediatric-to-adult-care-transitions-initiative/conditionspecific-tools) [Accessed January 2, 2020]

4. Barstow C, Shahan B, Roberts M: Evaluating medical decision-making capacity in practice. Am Fam Physician 98:40-46, 2018

5. Brown LW, Camfield P, Capers M, Cascino G, Ciccarelli M, de Gusmao CM, et al: The neurologist's role in supporting transition to adult health care: a consensus statement. Neurology 87:835-840, 2016

6. Christie D, Viner R: Chronic illness and transition: time for action. Adolesc Med State Art Rev 20:981-987, xi, 2009
7. Cunningham CT, Quan H, Hemmelgarn B, Noseworthy T, Beck CA, Dixon E, et al: Exploring physician specialist response rates to web-based surveys. BMC Med Res Methodol 15:32, 2015

8. Dore A, de Guise P, Mercier LA: Transition of care to adult congenital heart centres: what do patients know about their heart condition? Can J Cardiol 18:141-146, 2002

9. Flanigan TS, McFarlane E, Cook S: Conducting survey research among physicians and other medical professionals - a review of current literature, in Proceedings of the Survey Research Methods Section. Alexandria, VA: American Statistical Association, 2008, pp 4136-4147 (http:// www.asasrms.org/Proceedings/y2008/Files/flanigan.pdf) [Accessed January 2, 2020]

10. Flume PA, Anderson DL, Hardy KK, Gray S: Transition programs in cystic fibrosis centers: perceptions of pediatric and adult program directors. Pediatr Pulmonol 31:443-450, 2001

11. Gabriel P, McManus M, Rogers K, White P: Outcome evidence for structured pediatric to adult health care transition interventions: a systematic review. J Pediatr 188:263-269.e15, 2017

12. Got Transition: Measuring Transition. Washington, DC: Got Transition, 2019 (https://www.gottransition.org/ providers/leaving-measure.cfm) [Accessed January 2, 2020]

13. Hydrocephalus Association: Teens and Young Adults. Bethesda, MD: Hydrocephalus Association, 2019 (https:// www.hydroassoc.org/cause-view/teens-young-adults/) [Accessed January 2, 2020]

14. Lebrun-Harris LA, McManus MA, Ilango SM, Cyr M, McLellan SB, Mann MY, et al: Transition planning among US youth with and without special health care needs. Pediatrics 142:e20180194, 2018

15. Liu J, Gormley N, Dasenbrock HH, Aglio LS, Smith TR, Gormley WB, et al: Cost-benefit analysis of transitional care in neurosurgery. Neurosurgery 85:672-679, 2019

16. LoPresti M, Lam S, Orrico K, Browd SR, Ellenbogen RG, Martin J: Advocacy in pediatric neurosurgery: results from a 2017 survey of the American Society of Pediatric Neurosurgeons. J Neurosurg Pediatr 24:338-342, 2019

17. McDonagh JE, Kelly DA: Transitioning care of the pediatric recipient to adult caregivers. Pediatr Clin North Am 50:1561-1583, xi-xii, 2003

18. Rekate HL: The pediatric neurosurgical patient: the challenge of growing up. Semin Pediatr Neurol 16:2-8, 2009

19. Rothstein DH, Li V: Transitional care in pediatric neurosurgical patients. Semin Pediatr Surg 24:79-82, 2015

20. Sharma N, O'Hare K, Antonelli RC, Sawicki GS: Transition care: future directions in education, health policy, and outcomes research. Acad Pediatr 14:120-127, 2014

21. Simon TD, Lamb S, Murphy NA, Hom B, Walker ML, Clark EB: Who will care for me next? Transitioning to adulthood with hydrocephalus. Pediatrics 124:1431-1437, 2009

22. Spina Bifida Association of America: Guidelines for the Care of People with Spina Bifida. Arlington, VA: Spina Bifida Association of America, 2019 (https://www. spinabifidaassociation.org/guidelines/) [Accessed January 2, 2020]

23. Vinchon M, Dhellemmes P: The transition from child to adult in neurosurgery. Adv Tech Stand Neurosurg 32:3-24, 2007

24. White PH, Cooley WC: Supporting the health care transition from adolescence to adulthood in the medical home. Pediatrics 142:e20182587, 2018

25. Williams MA, van der Willigen T, White PH, Cartwright CC, Wood DL, Hamilton MG: Improving health care transition and longitudinal care for adolescents and young adults with hydrocephalus: report from the Hydrocephalus Association Transition Summit. J Neurosurg 131:1037-1045, 2019 


\section{Disclosures}

Dr. Hamilton is a consultant for Integra Canada.

\section{Author Contributions}

Conception and design: Martin, Rocque, Weprin. Acquisition of data: Martin, Rocque, Orrico. Analysis and interpretation of data: Martin, Rocque. Drafting the article: Martin, Rocque, Blount, Hopson. Critically revising the article: Martin, Rocque, Weprin, Blount, Hopson, Drake, Hamilton, Williams, White. Reviewed submitted version of manuscript: all authors. Approved the final version of the manuscript on behalf of all authors: Martin. Study supervision: Drake.

\section{Supplemental Information}

Previous Presentations

Portions of this work were presented at the 2019 American Society of Pediatric Neurosurgeons, Koloa, Kauai, HI, January 2019.

\section{Correspondence}

Jonathan E. Martin: University of Connecticut School of Medicine, Connecticut Children's Medical Center, Hartford, CT. jmartin03@connecticutchildrens.org. 